\title{
Reflets
}

Revue ontaroise d'intervention sociale et communautaire

\section{" Nager à contre-courant comme des saumons... " : les défis d'un centre de santé communautaire francophone en Ontario Entrevue avec Wesley Romulus}

\section{Michèle Kérisit}

Volume 1, numéro 2, automne 1995

La santé communautaire en Ontario français : défis et espoirs

URI : https://id.erudit.org/iderudit/026075ar

DOI : https://doi.org/10.7202/026075ar

Aller au sommaire du numéro

Éditeur(s)

Reflets : Revue ontaroise d'intervention sociale et communautaire

ISSN

1203-4576 (imprimé)

1712-8498 (numérique)

Découvrir la revue

Citer ce document

Kérisit, M. (1995). « Nager à contre-courant comme des saumons... " : les défis d'un centre de santé communautaire francophone en Ontario - Entrevue avec Wesley Romulus. Reflets, 1(2), 18-36. https://doi.org/10.7202/026075ar

Tous droits réservés (C) Reflets : Revue ontaroise d'intervention sociale et communautaire, 1995
Ce document est protégé par la loi sur le droit d'auteur. L'utilisation des services d'Érudit (y compris la reproduction) est assujettie à sa politique d'utilisation que vous pouvez consulter en ligne.

https://apropos.erudit.org/fr/usagers/politique-dutilisation/ 


\section{«Nager à contre-courant comme des saumons...»: les défis d'un centre de santé communautaire francophone en Ontario}

\section{Entrevue avec Wesley Romulus}

$D$ irecteur général du $C$ entre médico-sodal communautaire deT oronto

Installé en plein coeur de la ville, le C entre médico-sodal communautaire (cmsc) dessert depuis six ans la population francophone deToronto. $\mathrm{N}$ ous avons demandé à son direceur, W esley R omulus, de dresser le portrait du c MSC et de nous faire part des défis auxquels sont confrontés de tels centres $\mathrm{N}$ ous n'en sommes d'ailleurs pas restés là, car nous avions à coeur de parler aussi des succès...

L'entrevue que nous présentons id s'est déroulée davantage sous la forme d'une conversation que sous celle d' une entrevue formelle. $N$ ous savions que $M$. R omulus avait le verbe fadile et une connaissance approfondie du sujet. N ous voulions donc le laisser parler librement sur un sujet qui intéressera certainement les lecteurs de $R$ eflets

W esley $R$ omulus est bien connu des $F$ rancophones de l' $O$ ntario, non seulement à travers la fonction qu'il occupe actuellement mais aussi à cause de sa connaissance personnelle et variée de la francophonie ontarienne. T out d'abord enseignant à $H$ earst, il s'est ensuite «lancé dans l'administration» comme il le dit lui-même, en particulier dans des programmes fédéraux destinés aux jeunes. Par la suite, son travail pour l'A ssemblée des centres alturels de l'0 ntario l'a amené à parcourir la province, retrouver «ses amours d'antan», c'est-à-dire le nord de la province qu'il connaît bien, et la francophonie. C ette connaissance des spéafiatés régionales ontaroises, son engagement pour la «cause» francophone et son expérience de gestionnaire de terrain au $\mathrm{C}$ entre de $\mathrm{T}$ oronto le placent dans une position privilégiée pour 
nous parler des défis qu'un organisme destiné à promouvoir la santé communautaire en milieu minoritaire francophone peut rencontrer au cours de sa naissance et de son développement.

A joutons enfin que l'entrevue avec M. R omulus a eu lieu le 30 mai 1995, quelques jours avant l'élection du nouveau gouvernement conservateur de $\mathrm{M}$ ichael $\mathrm{H}$ arris. Bien que les coupures dramatiques qui s'annoncent dans les budgets sodaux et de santé risquent d'avoir déjà changé le paysage socio-communautaire franco-ontarien lors de la parution de ce numéro de $R$ eflets, nous avons estimé que les propos de M . R omulus gardaient toute leur pertinence, les centres de santé communautaire se retrouvant souvent au coeur du système de soins et de prévention qui se dessine.

\section{Michèle K érisit}

Professeure, É cole de service social, U niversité d'0 ttawa

MK : A vant de répondre à quelques questions sur la vie et les défis actuels $d u$ C entre, pourriez-vous nous raconter, en quelques phrases, l'histoire du CMSC. C omment est-il né, grâce à qui et pourquoi?

WR : Le Centre est né en 1989, mais sa période de gestation a commencé au début des années quatre-vingt. L'année 1989 a été l'aboutissement du rêve de certains leaders de la communauté qui ont eu pour vision la livraison de services en français, par des personnes parlant français. Cette vision a pris naissance au sein de la section régionale de Toronto de l'ACFO. Le projet s'est ensuite détaché de l'ACFO pour devenir celui d'un comité communautaire distinct qui, après une demande de subvention auprès du ministère de la Santé en 1988, a vu sa proposition budgétaire approuvée en décembre 1988. En mars 1989, je suis arrivé en tant que premier employé du CM Sc. À partir de là, nous avons commencé à mettre sur pied les services et les programmes, de sorte qu'en octobre de la même année, nous accueillions notre premier client.Voilà, dans les grandes lignes, I'histoire de la gestation et de la naissance du CMSc, qui a aussi coincidé, dans le contexte politique, avec la mise en place de la Loi 8 sur les services en français. 
M K : É tait-ce le premier $C$ entre de ce genre?

WR : Sans faire ombrage à la C linique Ste-Anne, à $O$ ttawa, dans la Basse-ville, nous sommes le premier C entre de santé communautaire multidisciplinaire francophone. $C$ 'est que la formule de la Clinique Ste-Anne n'est pas nécessairement une formule de santé communautaire, au sens propre. N ous sommes le premier centre de santé vraiment communautaire, multidisciplinaire, offrant toutes sortes de services sous le même toit, services qui ne sont pas livrés exclusivement par des professionnels de la santé.

M K : Q uels sont les services qu' offre le c m sc?

WR : $\quad$ N ous offrons des services dans plusieurs domaines mais on peut les regrouper sous trois chapeaux. Tout d'abord, les services de santé proprement dits: services curatifs de médecine, de soins primaires, de soins infirmiers, de soins psychologiques. $N$ ous offrons, par exemple, des services de diététique, des services et des programmes pour les mamans, les bébés, les femmes enceintes, les jeunes, les adolescents, etc.

Sous le second chapeau, nous regroupons les services à caractère social : services et programmes de psychothérapie, d'accueil, d'intervention en cas de crise. La dynamique de vie, la gestion de budget, la recherche d'emploi, les difficultés liées à la dépression et à l'adaptation sont quelques-uns des facteurs sociaux. N ous offrons aussi des services de dével oppement communautaire pour aider des groupes et des organismes communautaires à se mettre sur pied, ou à organiser des groupes de support.

Sous un troisième chapeau, se trouve le volet interprétation médicale francophone. $\mathrm{N}$ ous offrons des services d'accueil à Toronto et d'accompagnement dans les points de service de langue anglaise. C eci est nécessaire car nous n'avons pas toutes les spécialités. De plus, à l'échelle de la province, des régions comme celles de Timmins, Kapuskasing, C ochrane, Sudbury, ne disposent pas toujours de tous les services. Certaines spécialités ne se retrouvent qu'à Toronto. Les Francophones sont obligés de se déplacer et il n'est pas toujours possible de trouver des spécialistes qui parlent français dans des domaines très pointus comme le 
cancer, la chimiothérapie ou autre. $\mathrm{N}$ ous avons donc une équipe de professionnels qui accompagnent les personnes francophones afin de veiller à ce qu'elles comprennent toutes les opérations, les diagnostics, la procédure, les options, les alternatives, dans leur langue maternelle. Ils s'assurent également que le professionnel qui ne parle qu'anglais puisse comprendre les problématiques de son client de langue française.

MK: Vu le nombre de services que vous offrez, combien y-a-t-il d'employés?

WR : En ce moment, nous avons vingt- sept employés. En cinq ans, nous sommes passés de zéro à vingt- sept professionnels dans différents domaines qui offrent des services aux quarante-cinq mille francophones (et plus) àToronto.

M K : R evenons un peu sur la dientèle du c M sc, en partiaulier sur l'adaptation des pratiques des intervenantes et intervenants à la spédificté francophone. Comment adaptent-ils leurs pratiques aux spédificités de la population francophone deToronto?

WR : Au départ, nous avions un défi de taille à relever qui consistait à trouver des professionnels qui viendraient oeuvrer àToronto, en français. $\mathrm{N}$ ous avons posé cette problématique pour la première foisàToronto où nous avons demandé et activement recherché des professionnels parlant français. Tout le monde nous avait dit : «Vous perdez votre temps, vous n'aurez jamais personne.» La réalité a fait mentir ces pessimistes. Je ne pense pas que les professionnels aient eu à adapter leur pratique. Il y a eu, cependant, une période d'adaptation dans la mesure où ceux et celles qui livraient leurs services uniquement en contexte anglophone ont dû désormais écouter des problématiques, poser des diagnostics, élaborer des programmes et donner des ateliers en français. Je pense que la plupart de ceux et celles que nous avons embauchés pour ces postes, qu'ils aient été originaires de l'île $M$ aurice, d'H aïti, de $M$ artinique, du V ietnam, de France, de Belgique, des États $U$ nis, du Q uébec, de $\mathrm{H}$ earst, K apuskasing et j'en passe... , étaient contents de pouvoir oeuvrer dans leur langue maternelle. S'il y a eu une période de transition, ce fut surtout pour trouver et rassembler du matériel et de la documentation en français. 
N ous sommes en train de monter une banque de ressources grâce à la diligence de ces professionnels pour augmenter et prolonger leur efficacité. La période de transition pour passer de la langue de Shakespeare à celle de M olière s'est faite assez vite. Les problèmes que les gens présentaient étaient des problèmes humains. Alors, que ce soit en anglais ou en français, nos professionnels pouvaient s'identifier très vite aux problématiques qui leur étaient présentées.

M K : Q uant à la dientèle elle-même, pourriez-vous nous en résumer le profil? $Q$ ue vient-elle chercher au c $\mathrm{MSC}$ ?

W R : $\quad$ 'après nos recherches les plus récentes, la clientèle que nous desservons à l'heure actuelle est originaire de vingt- trois pays différents. Q u'elle soit née au C hili, en Espagne, en France, en $\mathrm{H}$ äti, au Zaïre, en Somalie... j'allais oublier les Arabes, les Égyptiens, les $M$ ahgrebins... son lien commun, c'est la langue française. $C$ es gens cherchent des services qui sont à leur portée, qu'ils comprennent et où ils peuvent s'exprimer. En effet, la plupart des gens ici sont techniquement bilingues: s'ils sont plombiers, par exemple, ou électriciens, ils peuvent très bien fonctionner dans la civilité de tous les jours: $\mathrm{H}$ ow are you? Fine thank you., etc. $D$ ans leur métier, ils parlent très facilement de pipe-wrenches, d'elbows. Pourtant, quand vient le temps de parler de leur foie, de leur vésicule biliaire, qui ne sont pas des termes qu'ils utilisent en anglais, il y a comme une «ratée» dans leur bilinguisme. Q uand vient le temps de parler de leurs émotions, de leurs sentiments, de leurs besoins en santé, ils se trouvent plus à l'aise en français et se disent : «Ah, quelqu'un me comprend, je ne suis pas obligé de chercher des mots, ou de deviner, ou d'inventer des expressions parce que je ne trouve pas le mot exact pour expliquer ce qui se passe entre la côte numéro cinq et la six.»

M K : E st-ce que vous avez I'occasion aussi d'offrir des services en arabe, par exemple, ou en somalien? Est-ce que vous avez choisi, aussi, cette option-là?

W R : $\quad$ N on, nos services sont offerts en français. A ux gens qui parlent anglais ou qui sont mariés avec des Anglophones, nous disons 
carrément que nos services sont disponibles en français. A lors si quelqu'un veut les obtenir en français, peu importe son autre langue, nous lui offrons ces services en français. C ette option est importante pour nous parce que nous sommes entourés d'une quinzaine d'autrescentressimilaires qui desservent lesAnglophones. $\mathrm{N}$ ous n'aurions pas vraiment de justification politique, sociale ou autre, à copier ce que les quinze autres font. $N$ otre spécificité réside dans la livraison de services en français et nous essayons de respecter ce mandat.

M K : A vec vingt-trois nationalités différentes, y a-t-il tout de même une population canadienne-française de souche qui fréquente le c M Sc?

W R : $\quad \mathrm{O}$ ui, bien sûr. C ependant, quand on fait le compte de toutes les personnes non de souche, elles sont plus nombreuses que les C anadiens-Français du Q uébec ou de l'O ntario, les FrancoO ntariens «pure laine». À Toronto, la proportion est en train de changer en faveur des gens nés ailleurs qu'au $C$ anada. Au début, notre clientèle était pluslargement constituée de Franco- $O$ ntariens ou de $C$ anadiens-Français d'origine. G raduellement, avec la popularité des services et du programme, nous avonsattiré une clientèle extrêmement diversifiée, cosmopolite et internationale.

M K : Pensez-vous qu'il existe des préocaupations de santé spéáfiques aux dientèles qui fréquentent le cm Sc?

W R : Certainesproblématiques que nous rencontrons ne sont sans doute pas spécifiques à la langue mais plutôt au fait que nous recevons une clientèle qui vient des tropiques. N ous avons, dans le domaine de la santé curative, par exemple, des cas de maladies tropicales. M ais, en général, la communauté francophone n'en est pas une qui a de graves problèmes de maladies industrielles, ou de plus graves problématiques que la moyenne. Les gens ne meurent pas de cancer plus vite que d'autres, ils ne sont pas atteints de sIDA plus que d'autres. 
M K : $\quad 0$ ffrez -vous des services qui concernent plus particulièrement les femmes, par exemple?

WR : $\quad O$ ui, nous avons des programmes comme $M$ aman et moi, nous offrons des cours de parentage que fréquentent surtout des femmes. N ous avons des cours pré-nataux, des cours post-nataux et nous offrons des programmes de soins aux bébés qui attirent beaucoup plus les mamans que les papas. N ous n'avons pas un volet spécifique à la santé des femmes, mais nous avons des programmes qui s'adressent uniquement aux femmes.

M K : A bordons plus en détail la philosophie de vos programmes. Vous vous appelez le $C$ entre médico-social communautaire $C$ e qui intrigue évidemment, c'est le rapport que vous établissez entre les trois mots: médical, social et communautaire. Q uelle est la part du social, du médical et du communautaire? Est-ce que les trois composantes, ou du moins les deux composantes sociale et médicale, sont étanches, ou est-ce qu'elles communiquent entre elles? Comment cela se passet-il, concrètement?

WR: II y a un énorme phénomène d'osmose entre les deux. II y a des parois poreuses entre nos services. $N$ otre philoso phie, notre mandat, c'est de promouvoir la santé globale des Francophones sur le territoire deToronto et de faciliter leur prise en charge. Puisque nous définissons la santé dans son sens le plus large, qui inclut la santé physique, psychologique, sociale, émotionnelle, et parce que nous nous targuons d'être le premier centre multidisciplinaire, nous capitalisons sérieusement sur l'aspect multidisciplinaire où l'individu n'est pas vu dans une optique étroite du genre «a psychothérapie et rien d'autre». N ous voyons l'individu dans son environnement, dans son contexte social, et nous essayons d'intervenir ou de le référer en prenant en considération sa globalité. Philosophiquement, nous n'avons pas une approche «pointue», mais plutôt une approche élargie. Les personnes que nous ne pouvons pas servir à l'interne, tant au niveau social qu'au niveau communautaire ou médical, nous essayons de les référer à des organismes qui vont pouvoir compléter notre gamme de services. 
M K : E t comment cela se passe $t-i l$ dans la pratique de tous les jours du centre et dans la mise en oeuvre quotidienne de cette approche globale?

W R : Prenonsle cas d'un homme battu qui arrive avec un oeil au beurre noir... N ous ne voyons pasque l'oeil au beurre noir, nous voyons au-delà du phénomène médical. II est en effet possible qu'il y ait des problématiques familiales qui expliquent cet oeil au beurre noir. À ce moment-là, les gens qui sont en charge des soins infirmiers ou médicaux vont s'occuper de l'oeil au beurre noir, mais vont aussi essayer d'aller plusloin en le référant pour une prise en charge par l'intervention sociale ou la psychothérapie, tout en respectant la confidentialité. Ils vont essayer de clarifier ce qui a provoqué ces incidents, avec le consentement de la personne. $\mathrm{N}$ ous ne truquons personne dans le processus. $\mathrm{N}$ ous établissons un climat de confiance qui permette aux gens de s'ouvrir. À ce moment-là, comme vous le voyez, un problème curatif conduit à une référence, toujours à l'interne, auprès d'intervenants en thérapie ou counselling familial. II est possible aussi qu'une telle situation nous amène à faire appel aux services de la Société de l'aide à l'enfance si l'on se rend compte que toute la famille, elle aussi, en a besoin... qu'il y a des petits «au beurre noir», que ce n'est pas seulement papa qui a l'oeil au beurre noir. Vous avez donc quelqu'un qui nous est arrivé lundi matin avec un oeil au beurre noir et que, mercredi, nous sommes en train d'aider en facilitant des solutions au niveau familial. Plus encore, on peut trouver qu'il est nécessaire de recourir à des services juridiques en français, services qui ne sont pas disponibles chez nous. N ous nous arrangeons alors pour référer la personne à des services juridiques à l'externe, en français, et nous essayons de lui faciliter les démarches. Donc, l'individu seul n'est pas en cause... nous avons vérifié cing yeux au beurre noir, mais nous avons vu une personne qui avait des besoins et recevait des services.

M K : E st-ce qu' une telle approche pourrait causer des défis particuliers au c M SC, tant aux niveaux organisationnel que financier?

WR : Les deux ministères qui nous subventionnent de façon primordiale, de façon abondante, sont tout à fait conscients que nous sommes un centre multidisciplinaire et que nous fonctionnons 
ainsi. U ne telle approche n'engage pas vraiment de dépenses supplémentaires. U ne fois établis les systèmes, les mécanismes d'échange entre professionnels et les protocoles, il y a très peu d'impact financier. Seule une augmentation dans le volume de services demandés exige parfois une augmentation du personnel ou un transfert de priorités. C'est plutôt au niveau de l'établissement de protocoles internes, de points de contact, de points de repère qu'une telle approche se met en place. Par exemple, tous les jeudis, l'équipe multidisciplinaire se rencontre et discute des cas de clients communs pour qui il faut prendre une décision dans la continuité des soins.

O n discute seulement de ce qui est nécessaire pour la prise de décision, et toujours dans la confidentialité et dans le respect de la personne. $\mathrm{N}$ ous nous assurons ainsi de la meilleure prise de décision possible, dans la mesure où chaque intervenant apporte un aspect, un éclairage différent. $0 \mathrm{n}$ pense ainsi respecter le mieux possible l'intérêt de la personne en question.

M K : Pensez -vous que, justement, cette approche disons médico-sodiale corresponde davantage au désir de la dientèle que vous avez? 0 u quel est le rôle de la dientèle dans la définition de ce genre d' approche?

WR : Au besoin, on consulte la clientèle. C elle-ci évalue le $C$ entre, les programmes et les services de façon ponctuelle et/ ou de façon formelle. Par sa présence, la clientèle sanctionne certaines choses qui se font bien et nous permet de valider ou d'améliorer, de modifier certaines choses qui ne se font pas aussi bien. Parfois c'est leur absence à certains programmes qui nous donne une indication de ce qui ne marche pas. Parfois nous utilisons un mécanisme d'évaluation formelle. M ais la clientèle, évidemment, n'ayant pas toute l'expertise voulue, ne peut pas être partout, à tous les paliers, à tous les niveaux, en train de décider de ce qui va se faire ou ne pas se faire. M ais là où la clientèle a son apport, celui-ci est apprécié, recherché, utilisé.

M K : La dientèle est-elle représentée au c M SC?

WR : A bsolument, avec un grand $A$, un grand $B$, un grand $S$... Parce 
que les membres de notre $C$ onseil d'administration ne se considèrent pas comme des bénévoles mais comme des représentants de la communauté. Les vrais propriétaires du C entre, ce sont les Francophones de Toronto, ce ne sont pas les ministères qui le subventionnent, pas plus que le personnel. La programmation répond aux besoins des clients. En bout de ligne, les propriétaires, ce sont ceux qui disent: «j'ai besoin de $x, y$ ou z.»

Jusqu'à présent, il y a eu, dans l'évolution du CM Sc, de 1980 jusqu'à 1994, beaucoup de travail en vase clos, de travail à l'interne, accompagné à l'occasion de validations par la clientèle. $M$ aintenant que les services sont établis, nous voulonsnous ouvrir davantage à la communauté et enrichir le volet communautaire. $\mathrm{N}$ ous $\mathrm{n}$ 'avons pas encore défini comment, mais la planification stratégique en marche va nous aiguiller sur certaines pistes vers un dialogue différent avec la communauté. L'élite qui avait prévu en 1980 de mettre les services sur pied avait le champ libre. À présent, cet espace se restreint en faveur d'une implication communautaire.

M K : $\quad$ N ous en arrivons donc au troisième volet de votre nom: le $C$ entre médicosocial communautaire. $D$ 'après ce que vous dites vous n' avez pas encore vraiment défini ce que vous appelleriez communautaire?

WR : II y a des défis qui nous attendent, premièrement l'établissement de partenariats communautaires beaucoup plus formels. En effet, pour le moment, nous avons établi des partenariats avec à peu près tous les organismes francophones, même anglophones, pour mieux desservir notre clientèle. C es partenariats ne sont cependant pas basés sur une philosophie qui a été pensée, établie et qu'on poursuit de façon acharnée et méthodique. N os relations, notre dialogue avec notre communauté, n'ont pas non plus été établis. $N$ otre publicité pour les rejoindre, le feedback dont j'ai parlé, ne sont pas suffisants. N ous voulons aller plus loin dans la recherche des besoins, la collecte des données, les études de marché. $\mathrm{N}$ ous voulons impliquer les Francophones beaucoup plus dans la prise en charge de leurs affaires, de leur santé. Pour ce faire, nos professionnels doivent alimenter le dialogue, pointer du doigt les domaines qui sont importants. 
U n autre défi qui nous attend dans ce même contexte concerne notre position en tant que Francophones à l'égard des réformes en cours. $L$ à, I'O ntario a décidé de réformer à peu près tout ce qui est réformable et, que ce soit louable ou non, cela se fait. Les Francophones ne sont pas présents dans ces réformes-là, ils sont absents des comités directeurs, des comités de consultation, des groupes focus ou même des listes de distribution de l'information initiale. C'est tout un défi pour nous, avec les maigres moyens du bord, de nous assurer que premièrement, les Francophones soient au courant de ce qui est réformé, et deuxièment qu'ils connaissent les enjeux et les façons de se mobiliser, où frapper, où avoir un impact. C'est tout un défi pour nous de pouvoir mobiliser notre communauté pour qu'elle soit efficace dans ces réformes, pour qu'elle prenne la place qui lui revient.

M K : Pour ce qui est des réformes des soins de santé, que l'on dirait sans fin, est-ce qu' aduellement vous en voyez l'impad sur le $C$ entre?

WR : II n'y a pas eu d'impact encore, parce que je pense que la réforme, la restructuration des hôpitaux se préparent. Si certains hôpitaux ferment, certains soins seront relégués à la communauté. C omment cela sera-t-il fait? Avec des sous, sans sous? Avec des paroles, des promesses? Et avec du chantage?Tout est possible, mais ce $n$ 'est pas encore fait. C haque hôpital en est encore à dire: $« O$ ui, nous sommes d'accord pour la coupure, mais pas nous, coupez le voisin.» Chacun dit cela à tour de rôle; c'est la chaise musicale du roi Arthur. De plus, le gouvernement fédéral menace de couper les transferts de paiement aux provinces qui, en retour, ou bien vont prélever plus de taxes ou bien vont couper nos subventions. C'est à ce troisième niveau que nous allons surtout sentir l'impact. C e n'est pas encore arrivé, mais on sent que la chaleur monte.

M K : $\quad E$ st-ce que le $C$ entre se prépare d'une certaine façon à ces grands change ments?

WR : N ous avons entrepris une opération de diversification de notre financement (que c'est bien dit !... ). N ous pensons à la création 
d'une seconde corporation, parallèle au cm Sc, mais avec qui elle aurait des affinités. $C$ ette corporation aurait les mains libres puisqu'elle ne serait pas subventionnée par les ministères. Les fonds qu'elle recueillerait pourraient être utilisés par le cmsc pour combler les coupures qui, nous le savons, viendront. II y a donc prise de conscience: nous devons nous positionner financièrement pour ne pas être estropiés, handicapés.

M K : Pensez-vous justement, dans œ domainelà, que le fait que vous soyez un centre de santé communautaire puisse vous donner des atouts pour survivre, ou même mieux vivre?

W R : Comme je l'ai dit tout à l'heure, il peut y avoir dévolution des responsabilités des hôpitaux et institutions vers la communauté. Tout le monde va applaudir, parce que notre crédibilité en prend de l'importance. «N ous sommes fantastiques, pourra-t-on dire, tout le monde nous fait confiance» C ependant, cela se compare à adopter cinq enfants d'un coup: si vous n'avez pas les moyens financiers d'absorber le coût de cinq enfants de plus dans une famille de deux personnes, ce geste noble, ce coup de coeur, peut conduire à des difficultés. Si cette dévolution des responsabilités des institutions vers la communauté n'est pas accompagnée de moyens financiers concrets, c'est la mort des services communautaires. Vous faites asseoir un éléphant sur un petit écureuil et c'est fini, il est aplati. $\mathrm{N}$ ous devenons alors victimes de notre succès, victimes de notre crédibilité, et aussi victimes, peut-être, de l'incurie de nos dirigeants gouvernementaux. Je ne présume pas qu'ils soient comme ça, mais cela ne me surprendrait pas. D'autres l'ont fait. Faire des promesses vides, prendre des décisions qui ont des répercussions financières non respectées, cela peut être un danger réel. O n l'a constaté dans le domaine de la santé mentale où les institutions psychiatriques sont appelées à fermer et à renvoyer les gens dans la communauté, pour mener une vie aussi normale que possible. Bravo! Félicitations! Excellent! Q uelle vision! C ependant, cela fait quatre ans que nous demandons de l'argent supplémentaire pour embaucher desintervenants pour travailler avec ces personnes dans la communauté. $\mathrm{N}$ ous pouvons avoir accès à la formation, nous avons le 
cadre d'opération, nous avons tout ce qu'il faut: la population, les clients, mais le ministère refuse de donner les subventions. 0 n évoque toutes sortes d'excuses et de subterfuges pour donner ces argents aux centres anglophones déjà existants. LesFrancophones, quant à eux, se heurtent toujours au même refus. $D$ ans ce grand geste magnanime de fermeture d'institutions et de rejet dans la communauté d'individus, les Francophones se retrouvent doublement en peine.

M K : Et en partie à cause d'obstades qui se trouvent à un niveau politique, gouvernemental...

W R : Administratif, bureaucratique, oui.

M K : V ous venez d'évoquer les défis qui se posent à des centres communautaires de santé, mais avec la double caractéristique, évidemment, d'être francophone, d'être à l' autre bout du bâton.

WR : Le bout ignoré du bâton. Souvent, je pense qu'il y a un peu de mauvaise foi, quand ce bout est ignoré.

MK : Q u'est-ce que vous voulez dire par là?

WR : C'est que nous avons, en 0 ntario, des conseils régionaux de santé. C es nobles institutions sont indépendantes du ministère et sont donc hors de l'impact politique que les Francophones sont habitués à avoir auprès des ministères, où ils doivent être, malgré tout, considérés comme des citoyens et des contribuables à part entière. C es organismes de planification ignorent cependant systématiquement les Francophones, que ce soit dans la région de Toronto, d'Algoma, de Cochrane ou peu importe le district, dans une région désignée, incluant $M$ ississauga, entre autres. S'il n'y a aucun élément francophone dans ces organismes qui planifient la distribution des fonds et donc des services, qui approuvent et envoient au ministère ce qui est consacré, il n'y aura jamais rien de francophone de consacré, de béni, ni de véritables retombées. 
MK : Et vous pensez que ces «silences», ces «oublis» partent d'une volonté politique?

WR : O ui, oui, oui, oui.

M K : N e pourrait-on pas y voir d'autres raisons: apathie ou diffiaulté de s'organiser en tant que $\mathrm{F}$ rancophones en 0 ntario, étant donné la diversité sociale, régionale, et maintenant alturelle des $\mathrm{F}$ ranco- 0 ntariens?

WR : Vous souvenez-vous de votre question préliminaire quand vous me demandiez quel était l'impact d'un centre communautaire dans une communauté quelconque? C'est un impact de catalyseur. Q uand vous avez des professionnels qui livrent des services avec des moyens inadéquats, qui mettent l'eau à la bouche des clients en leur montrant qu'ils peuvent avoir accès à des soins et des services de qualité, dans leur langue, à leur portée, les gens se demandent s'ils ne pourraient pas, justement, en obtenir davantage. C'est à ce moment-là que les professionnels, qui sont aussi en contact avec ce qui se brasse dans les ministères, dans différents départements, au sein de différents projets et initiatives, peuvent mettre ces informations à la portée de la clientèle. Sans les centres, les gens ne peuvent pas savoir ce qui se trame dansles réformes. D emandez par exemple à quelqu'un dans la rue ce qu'est la réforme de la pharmacologie; il ne pourrait pas vous répondre. Par contre, si vous mettez en place un système de santé communautaire, là, les diététistes, les infirmières peuvent traduire pour les gens ce que représente une telle réforme. Les centres de santé communautaire ont un effet catalyseur. Ils offrent des services, bien sûr, mais ils véhiculent aussi de l'information. Ils sensibilisent le public et réveillent les consciences. Ils ont aussi un rôle de revendication, parce qu'en ayant recueilli les besoins de la clientèle, ils peuvent aller maintenant frapper à certaines portes et dire: «nous savons que les besoins sont $x, y, z$.» Si elle n'a pas cet outil de développement, au niveau de la santé, du développement social et communautaire, une communauté est plus démunie qu'une autre. 
M K : Pour continuer à parler du contex te politique dans lequel peut naître et se développer un centre de santé communautaire, posons la question de la réussite et du succès. É tant donné votre expérience personnelle de directeur de centre, et étant donné les diversités régionales que vous connaissez, quels ont été, à votre aviş les instruments de réussite du C M Sc? B eaucoup de centres sont en train de se mettre en place. Q uels seraient les instruments de réussite que vous choisiriez de partager avec ces organismes qui n'en sont qu'à leurs débuts?

W R : Sans être prétentieux, il faut d'abord, pour connaitre un succès à long terme, que les besoins de la clientèle soient identifiés avec le plus grand soin possible. À ceci j'ajoute une volonté, un courage de la part des Francophones pour défendre leurs points de vue, parce qu'ils nagent un peu comme des saumons, à contre-courant, le courant qui valorise l'uniformité à l'anglo- saxonne. «Puisque vous êtes en 0 ntario, vous devez parler anglais: Everything is available, why don't you speak E nglish?» Or nous venons contrecar rer tout cela, avec des services en français. II faut une volonté politique de la part des personnes engagées à défendre leur point, à se battre, à frapper aux portes, à se faire entendre, à se faire dire non et revenir à la charge. II faut s'allier des professionnels convaincus, des gens qui sont presque des missionnaires, des gens qui y sont pour la cause autant que pour la satisfaction personnelle; bref, des gens qui croient dans le phénomène francophone, qui croient à sa survie et qui sont prêts à y mettre du 110, 115, $120 \%$. II faut également mettre sur pied des services de qualité, qui ne vont pas décevoir les gens. La qualité des services est une valeur essentielle pour que les gens y aient recours. II faut adopter une attitude qui mette en valeur l'accessibilité des services: malgré toute notre compétence, tous nos jargons, nos diplômes et notre préparation, il faut se mettre au niveau des gens, les mettre à I'aise pour qu'ils se sentent accueillis tels qu'ils sont. II faut aussi constamment évaluer, remettre en question ce qu'on fait, de façon à s'ajuster, à se repositionner, à se modifier.

M K : N e serait-il pas important de mentionner id l'accessibilité des services à des dientèles très diverses, telles que vous l'évoquiez au début de notre 
conversation, accessibilité qui doit aussi atteindre les minorités au sein même de la minorité franco-ontarienne?

WR : Là où il y a demande, il faut absolument que la diversité de la communauté desservie soit reflétée dans le personnel. Sinon, un pourcentage ne croira jamais à l'accessibilité du centre. Si vous avez uniquement des Q uébécois «de souche» comme membres du personnel - notez bien que je n'ai rien contre les Q uébécois - vous pouvez annoncer à tort et à travers que vos services sont diversifiés, un pourcentage de la population n'y croira pas.Vous embauchez une personne de couleur différente des Q uébécois blancs et là, tout d'un coup, une autre clientèle va venir, qui se sent à l'aise, qui n'a même pas besoin de parler à cette personne, mais qui sent que c'est un milieu où il y a du semblable à soi. Et lesgens sont attirés par le semblable... LesFrancophones viennent ici parce qu'il y a du semblable au point de vue linguistique. Les Zaïrois viennent parce qu'ils savent qu'au Centre, il y a quelqu'un du $\mathrm{Z}$ aïre, les $\mathrm{H}$ aitiens viennent parce qu'il y a des $\mathrm{H}$ aitiens dans le personnel, et c'est la même chose pour les gens duV ietnam, de la M artinique, de la Suisse, etc. Parce qu'ils sentent, ils entendent un accent qui leur est familier, ils se sentent un peu chez eux. Donc, là où c'est exigé, il faut le faire. À Sault-Ste-M arie, ce n'est peut-être pas le cas, car ce n'est pas Toronto, mais 0 ttawa, par exemple, pourrait suivre ce modèle-là, parce que la diversité est là. II faudrait toujours être sensible et refléter cette diversité tant au niveau des représentants qu'au niveau du personnel qui livre des services. Les gens ne vous diront jamais: «je ne viens pas souvent parce que je ne suis pas à l'aise.» $M$ ais les gens viendront s'ils sont à l'aise. Et cela contribue positivement à la réussite d'un centre comme le nôtre. Personne ne vous dira: «C 'est parce que vous avez seulement des $\mathrm{N}$ oirs comme personnel que je ne viens pas.» $\mathrm{M}$ ais si vous mettez deux ou trois B lancs parmi les $\mathrm{N}$ oirs, vous allez voir, ça va marcher...

M K : $\quad$ D 'où, encore une fois, la nécessité d' identifier les besoins de la population.

W R : $\quad$ N ous avons fait, par exemple, un dépistage anonyme, parce qu'il y en avait un réel besoin parmi les Francophones. $N$ ous en faisons dans nos deux points de service, ici et à Fairview. N ous 
avons des gens qui sont formés spécialement pour faire ce genre d'enquête. C ertaines personnes nous demandent parfois de mettre sur pied de nouveaux programmes, mais nous ne le faisons pas si nous n'en percevons pas le besoin dans la population que nous desservons. Par contre, nous initions parfois des projets parce que nous savons que ce besoin existe, même s'il n'est pas clairement formulé. Par exemple, nous avons fait des déclarations contre l'excision clitoridienne, parce que nous avons des clientes qui vivaient dans ce contexte-là. $\mathrm{N}$ ous avons sensibilisé notre personnel à toutes ces coutumes, ces pratiques, comment ça se fait, pourquoi ça se fait, etc. $N$ ous sentions qu'il y avait un besoin. Si on perd contact avec la commuanuté, on devient élitiste, et on meurt.

MK : Y a-t-il un programme, un projet ou un service du c m sc que vous voudriez partager avec les lecteurs, parce qu'il est innovateur et parce que vous en êtes particulièrement fier?

W R : Peut-être est-ce notre photorama A ssez. D ans nos efforts de lutte contre la violence, nous avons conçu et réalisé un photorama intitulé A ssez! Ce photorama n'existe dans aucun autre centre. $D$ 'ailleursil est maintenant rendu en France, en Suisse, en Belgique et les gens peuvent le copier partout. II reprend trois petites vignettes représentant différentes situations de violence. Le ministère nous a demandé de le traduire en lingala pour les communautés zaïroises qui comprennent le lingala et en créole pour les communautés haïtiennes. $\mathrm{C}$ e fut un projet unique et inédit.

M K : Et comment utilisez-vous le photorama? C omme base de départ pour une animation?

W R : $\quad 0$ ui. Le ministère nous a demandé d'aller au-delà de notre mandat local et de faire une tournée provinciale. $N$ ous avons établi un partenariat avec un autre organisme à mandat provincial et nous avons offert des ateliers dans beaucoup de régions désignées, nous y avons formé des gens du milieu, des enseignants, des enseignantes, destravailleurs sociaux, pour qu'ilsutilisent A ssez! comme 
outil d'animation et puissent développer des ressources locales répondant aux besoins spécifiques des gens. Les besoins d'un refuge pour femmes àToronto ne ressemblent pas à ceux de Pénétang ou de $\mathrm{H}$ awkesbury, par exemple. $\mathrm{D}$ ans la formation des animateurs et animatrices locales, nous nous sommes arrangés pour que les gens comprennent la nécessité d'aller chercher sur place leurs propres ressources.

M K : E $n$ résumé, quels sont les défis particuliers qui s'offrent à un organisme de santé communautaire francophone comme le vôtre?

W R : Je parle évidemment de la position d'un centre qui est déjà établi. Le défi de ce centre est différent du défi, par exemple, qui se pose à la communauté deW indsor, qui essaie d'avoir un C entre qu'il n'aura sans doute pas. Le défi, pour nous, c'est de nous frayer un chemin à travers la bureaucratie, à travers la volonté politique. Faire reconnaitre le fait français constitue le défi majeur. U ne fois le fait français reconnu et accepté, le reste, la compétence, la mise sur pied, n'est pas un problème pour les Francophones. C'est cette reconnaissance initiale qui est extrêmement importante. À Kapuskasing où on est en train de mettre sur pied un centre, le défi est peut-être différent. M ême chose pour C ornwall, Sudbury, $\mathrm{H}$ amilton-Welland ou le Témiskamingue. La communauté du Témiskamingue rencontre d'ailleurs une opposition extraordinaire Tant que le Centre n'en était qu'à l'état de projet, lesAnglophones, les médecins, les hôpitaux disaient: «Laissez-les donc, les Francophones, ils se réunissent, ce n'est pas grave.» Le ministère a accordé un budget; leT émiskamingue va donc avoir son centre de santé communautaire francophone. La première réaction a été: «O $n$ doit empêcher ça, ça se peut pas, ça ne doit pas.» $C$ 'est de ce genre de choses dont je parle. À T immins, c'est pareil. La communauté francophone avait demandé un centre; on lui a donné 200000 \$ pour faire un petit projet de santé, pour publier des petits dépliants et informer la population sur certains sujets. Les gens se sont seulement nommés «C entre de santé communautaire de T imminss et le Conseil régional de santé, de complicité avec le ministère de la Santé, leur a dit qu'ils ne pouvaient 
pas faire cela, qu'ilsn'étaient pas un C entre et ne pouvaient donc pas s'appeler ainsi. C ela a écrasé bien des enthousiasmes. C'est cette volonté politique, c'est ce marasme bureaucratique qui est le plus grand défi posé aux Francophones. S'ils avaient été reconnus, avaient été invités à s'asseoir aux tables de concertation et de décision, au même titre que les A nglophones, cela aurait été différent. $M$ ais nous frappons à la porte, on n'ouvre pas, nous frappons, nous donnons des coups de pied, nous crions, on n'ouvre pas. Alors nous devons prendre les marteaux-pilons, la dynamite, enfin, quelque chose!

MK : Tant que ça?

W R : C'est ça, pas la bombe, quand même. 